\title{
Investigation of Pyrolyzing Ablators Using a Gas Injection Probe
}

\author{
N. C. Martin*, C. C. Tillson, J. M. Meyers ${ }^{\dagger}$ and D. G. Fletcher ${ }^{\ddagger}$ \\ University of Vermont, Burlington, VT \\ D. Dang ${ }^{\S}$ and I. Boyd ${ }^{\top}$ \\ University of Michigan, Ann Arbor, MI
}

\begin{abstract}
The pyrolysis mechanism of Phenolic Impregnated Carbon Ablator (PICA) makes it a viable candidate for thermal protection systems for spacecraft atmospheric entry. However, a better understanding of the pyrolysis mechanism and of the interaction of the pyrolysis gases with the plasma flow is needed to improve heat-shield designs. The present study extends previous work in the design, development, and testing of a gas-injection probe to simulate pyrolysis in the UVM 30kW Inductively Coupled Plasma (ICP) Torch Facility. A parallel effort in Computational Fluid Dynamics (CFD) modeling of the injection probe during plasma testing is also discussed. Measurements were obtained with the injection probe in the facility in an argon buffered nitrogen condition with carbon dioxide being injected into the flow through a FiberForm plug. Spatially resolved high-resolution emission data were acquired during this test. Spectral signatures of $\mathrm{CN}, \mathrm{OH}$, and $\mathrm{NH}$ bands were detected and their spatial locations determined using a spectrometer equipped with a CCD camera at the exit plane. At the same time the relative conditions in the facility were used to create a CFD model of the experiment. Comparisons between the measured, spatially resolved, emission spectra and the simulations calculated using a radiative transport model from the CFD results show reasonable agreement.
\end{abstract}

\section{Introduction}

Phenolic Impregnated Carbon Ablator (PICA) based heat shield materials release gas, in a process called pyrolysis, as the binding resin sublimates when the internal temperature increases. Pyrolysis sublimation and gas decomposition as it passes through the charred internal structure of PICA are current research topics that are under active investigation. While such studies will provide much needed information about the internal processes of a pyrolyzing ablator, further investigation is needed to understand how the released gases interact with the superheated plasma produced when a spacecraft is entering an atmosphere.

Investigation of pyrolysis gases has been undertaken in an extension of earlier work. ${ }^{4}$ This investigation focuses on the interactions of PICA pyrolysis gases with different plasmas using emission spectroscopy to measure the characteristics of the pyrolysis chemistry. To better understand the interactions between the pyrolysis gases and plasma, a gas injection probe has been designed for use in the UVM $30 \mathrm{~kW}$ Inductively Coupled Plasma (ICP) Torch. This probe allows for the injection of controlled amounts of simulated pyrolysis gas directly into the plasma flow. Using the capabilities of the gas injection probe, the interactions between the injected gas (simulating the pyrolysis gas) and the plasma can be studied and analyzed. The long run times of a simulation also allow for extensive laser diagnostics and emission data to be collected. A parallel modeling effort using advanced computational models is also underway. This paper reports on the combined effort, which provides a basis for the development and application of the appropriate laser spectroscopic techniques. Previous work on pyrolysis simulation has been done in the UVM Plasma Lab with an earlier

* Graduate Student, Mechanical Engineering Program, AIAA Student Member.

${ }^{\dagger}$ Research Assistant Professor, Mechanical Engineering Program, Senior AIAA Senior Member.

‡Professor, Mechanical Engineering Program, AIAA Associate Fellow.

$\S$ Graduate Student, Aerospace Engineering, AIAA Student Member.

๑ Professor and James Knott Chair, Aerospace Engineering, AIAA Fellow. 
version of a pyrolysis injection probe. ${ }^{3}$ Although this work produced useful results, several shortcomings were identified in the design of that probe. These shortcomings included difficult thermocouple access to the back of the test sample, no dedicated pressure tap to measure the pressure of the injectant gas, and the design of the probe was extremely complex leading to time consuming repairs if anything failed.

The probe that is the subject of this paper has been designed to solve most of the shortcomings of the earlier probe as stated above. It is large enough to easily handle multiple thermocouples as well as other internal instruments. The probe has a port for gas injection as well as one for for a pressure tap. Multiple different custom sample holders can be used with the probe's universal mounting system. Cooling for the device is provided by the closed loop cooling system. The remainder of this paper covers the UVM $30 \mathrm{~kW}$ (ICP) Torch Facility, the probe design, results, a discussion of the computational simulations, and future work that is will be done using the probe.

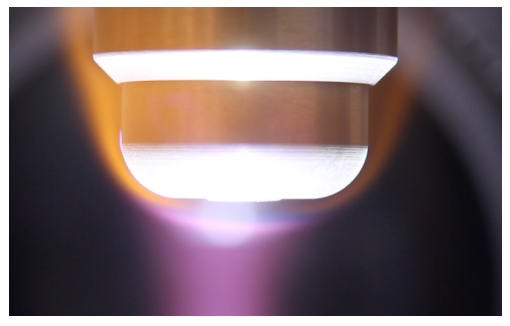

Figure 1: Image of $.86 \mathrm{~g} / \mathrm{min}$ of $\mathrm{CO}_{2}$ being injected into a soft Nitrogen plasma.

\section{UVM $30 \mathrm{~kW}$ ICP Facility}

All experiments were performed within the UVM $30 \mathrm{~kW}$ ICP Facility which was designed to simulate the near surface chemically reacting boundary layer of atmospheric entry and hypersonic flight. ${ }^{2}$ The use of ICP-type facilities for entry and hypersonic testing applications is not new, but in the U.S. arc-heated facilities are more commonly used for these investigations. However, in arc-heaters the direct arc attachment to electrodes produces molten copper as a stream contaminant. Copper atom emission lines are strongly evident and raise questions about the flow chemistry. In an ICP torch, heating is done through electron excitation via magnetic field coupling, thus providing a contaminant-free flow. Replication of the flight trajectory boundary layer (Fig. 2) is achieved when boundary layer edge velocity gradient, enthalpy, and total pressure of the subsonic ICP facility are matched with those of the flight condition.
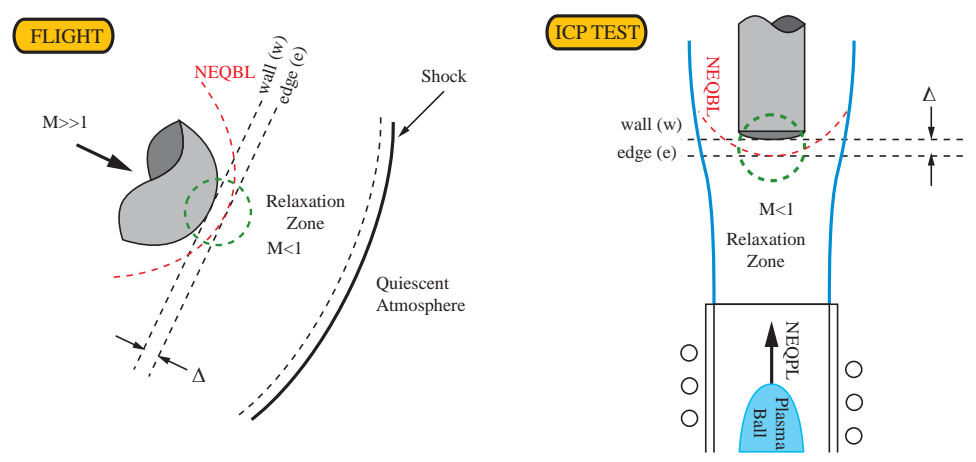

Figure 2: Illustration of reentry boundary layer (shown with thickness $\Delta$ ) in an inductively coupled plasma facility.

The UVM $30 \mathrm{~kW}$ ICP Torch Facility (see Fig. 3) is capable of providing chemically pure Air, $\mathrm{N}_{2}, \mathrm{O}_{2}$, $\mathrm{CO}_{2}$, and Ar plasmas (or mixtures thereof) at trajectory relevant conditions. The plasma jet is currently defined by a $36 \mathrm{~mm}$ inside diameter quartz tube. Normal facility operating conditions are given in Table 1 . At the plasma ball location in the induction zone the temperature is on the order of 10,000 K. The subsonic plasma travels vertically upward, exiting the quartz tube as a free jet that cools as it evolves toward a local 
thermodynamic equilibrium (LTE) state. For air plasma tests there is, as yet, no evidence that the plasma is not in LTE, while for nitrogen plasma, spectrally resolved emission measurements indicate a degree of nonequilibrium. ${ }^{5}$

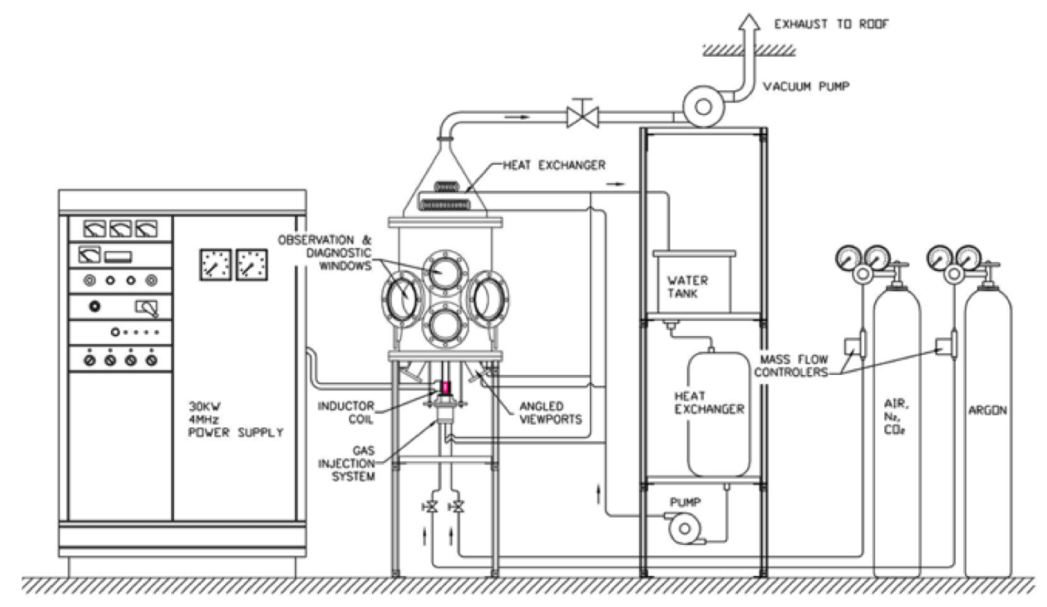

Figure 3: Schematic of the UVM 30kW ICP Torch Facility. The power supply, gas injection system, test chamber, and water cooling system can be seen. ${ }^{2}$

\begin{tabular}{cc}
\hline \hline Test Gas & $\mathrm{Ar}, \mathrm{N}_{2}, \mathrm{O}_{2}, \mathrm{Air}, \mathrm{CO}_{2}$ \\
Maximum Power & $30[\mathrm{~kW}]$ \\
Normal Operating Pressure & 100 to $200[\mathrm{torr}](13$ to $26[\mathrm{kPa}])$ \\
Stagnation Heat Flux & 10 to $150\left[\mathrm{~W} / \mathrm{cm}^{2}\right]$ \\
Mach Range & 0.3 to $1.4[-]$ \\
Plasma Jet Diameter & $36[\mathrm{~mm}]$ \\
Operating Frequency & 2 to $3[\mathrm{MHz}]$ \\
\hline \hline
\end{tabular}

Table 1: UVM $30 \mathrm{~kW}$ ICP test parameters.

\section{Experimental Arrangement}

\section{A. Probe Design}

The new injection probe design is based off the goose-neck probe that is currently used in the lab. ${ }^{2}$ The new probe is an upscaled version of this probe and therefore has more internal room to fit thermocouples and the gas injection plumbing. A custom end cap was machined for the end of the probe with a threaded design in order to facilitate the attachment of many different sample holders as well as provide a seal to keep the cooling water inside the device. This adapter also has an o-ring groove on its front face in order to provide a sealed volume for the injectant gas. A CAD image of this can be seen in Fig. 4a. A threaded collar was also made to keep the sample holder in place on the face of the adapter and provide the pressure to seal the holder against the o-ring. A drawing of the complete end cap assembly can be seen in Fig. 4b.

The new probe has the ability to translate vertically within the test chamber. It can be lowered until the sample is flush with the base of the facility or, with the current sample holder, raised to a height of $95 \mathrm{~mm}$ above the base. This maximum height has been extended further with a new adapter plate for the upper viewport window of the test chamber. This will allow the sample to be placed at about 160 to 220 $\mathrm{mm}$ above the base of the facility. The probe also has the ability to rotate once put in place. This feature, along with the two forty-five degree bends incorporated into the design, allow the probe to be rotated out of the plasma flow until it is needed. The rotation also allows for the proper test conditions to be achieved in the facility before exposing the sample to the plasma. A complete labeled diagram of the new probe can be seen in Fig. 5 . 


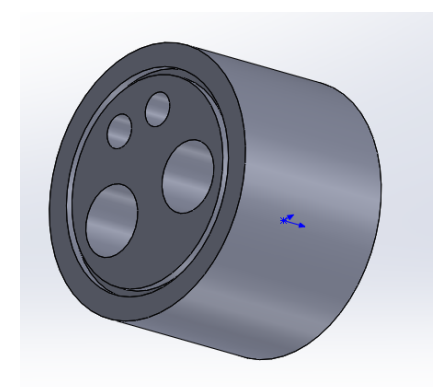

(a)

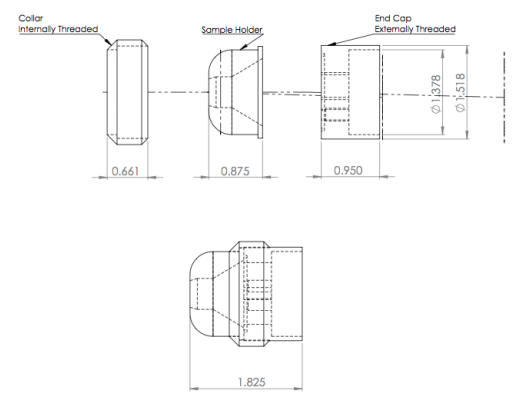

(b)

Figure 4: Shows the end cap (a) and the sample holder and end cap assembly (b).

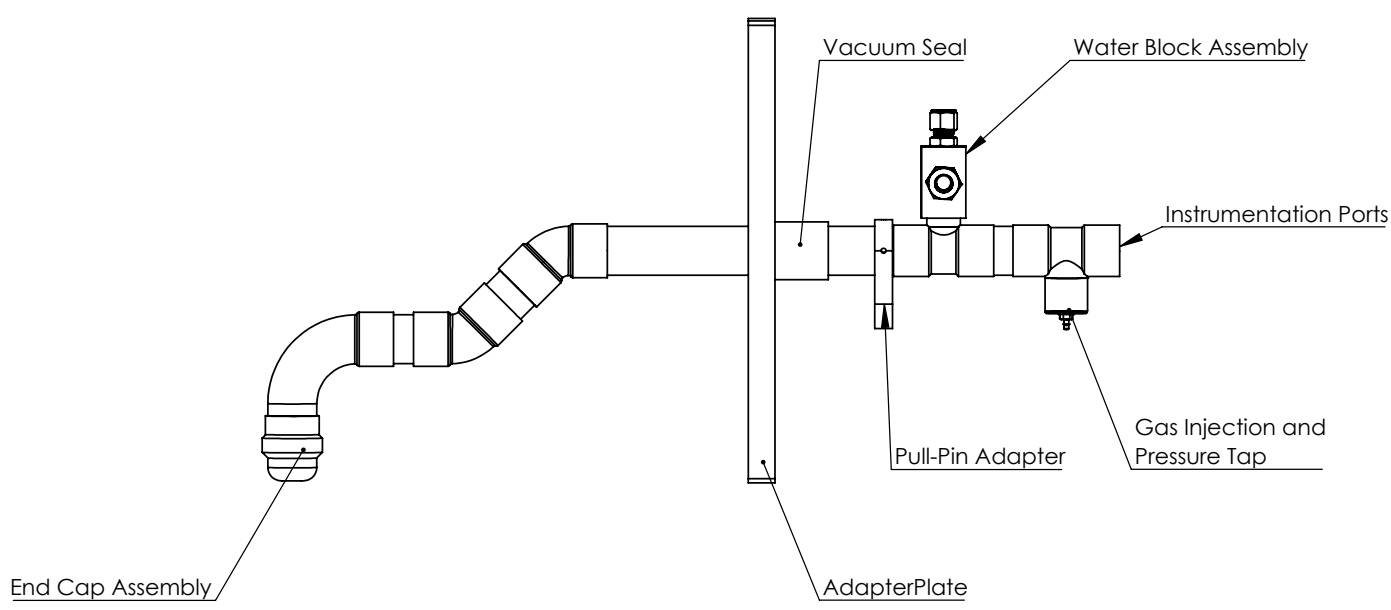

Figure 5: Shows the overall labeled configuration of the probe.

Thorough testing of this probe has lead to the conclusion that it does not cool the brass sample holder well enough to allow for long run-time testing or testing in higher enthalpy conditions such as pure Nitrogen. The lack of long run-time testing also means that LIF based measurements can not be taken using this probe. Steps have already been taken to try and solve this issues and are discussed below in Section V.

\section{B. Instrumentation}

A Canon EOS 7D DSLR camera was used to record video for all tests as well as take photographs. These recordings and photos enabled us to see and study the injectant gas and how it is interacting with the plasma as it enters the flow. A Princeton Instruments IsoPlane320 with a PIXIS:256 imaging CCD was be used to take measurements of the species present in the flow as well as other spectral data. The collection region was imaged onto the vertical entrance slit of the spectrometer using a z-fold arraignment. The assembly included a $250 \mathrm{~mm}$ concave mirror where $\mathrm{d}_{0}$ is $736.6 \mathrm{~mm}, \mathrm{~d}_{i 1}$ is $190.5 \mathrm{~mm}$, and $\mathrm{d}_{i 2}$ is $330.2 \mathrm{~mm}$. In this configuration the image height $h$ that is imaged onto 256 vertical pixels of the CCD is $9.4 \mathrm{~mm} .{ }^{4}$ The optical arraignment, including the collection beam path, and the vertical collection region can be seen below in Fig. 6. Table 2 shows the complete specifications of the IsoPlane spectrometer. 


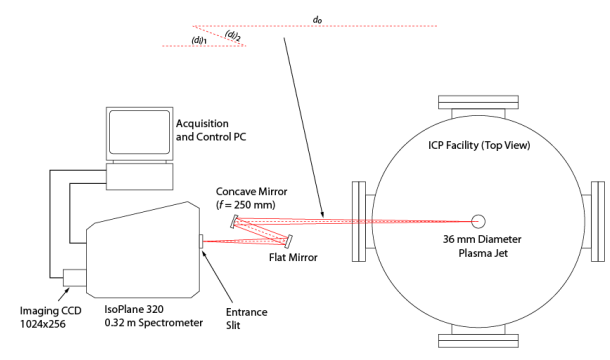

(a) IsoPLane Experimental Set-Up

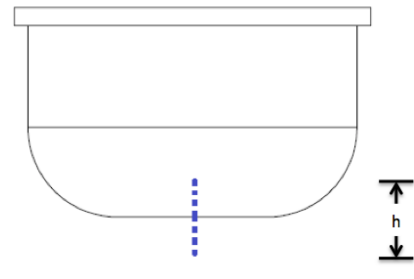

(b) Vertical Collection

Figure 6: Shows the IsoPlane Set-Up (a) and the Vertical Collection area (b). ${ }^{4}$

\begin{tabular}{lc}
\hline \hline Wavelength & See Grating \\
Slit Width & $5-10 \mu \mathrm{m}-3 \mathrm{~mm}$ \\
& $600 \mathrm{G} / \mathrm{mm}$ ruled $-300 \mathrm{~nm}$ blaze \\
Grating & $600 \mathrm{G} / \mathrm{mm}$ ruled $-500 \mathrm{~nm}$ blaze \\
& $900 \mathrm{G} / \mathrm{mm}$ Holographic - NIR Optimized \\
& $0.142 \mathrm{~nm}(300 \mathrm{~nm}$ blazed $)$ \\
$F W H M$ & $0.140 \mathrm{~nm}(600 \mathrm{~nm}$ blazed $)$ \\
& $0.09 \mathrm{~nm}(\mathrm{NIR}$ optimized $)$ \\
Resolution & PIXIS $1024 \times 256(26 \mathrm{~mm} \times 6.7 \mathrm{~mm})$ \\
& element imaging CCD \\
\hline \hline
\end{tabular}

Table 2: IsoPlane Specifications ${ }^{4}$ 


\section{Results and Discussion}

\section{A. ICP Experiments}

Due to facility and probe issues only one data set was taken for a gas injection condition. An image of this data can be seen in Fig. 7. The IsoPlane and Pixis camera are currently uncalibrated so the data is therefore given in intensity units. In the large plot, the $\mathrm{x}$-axis defines the wavelength and the y-axis the location on the vertical sample region. The smaller top plot shows the spectrum distribution at a single location. In this case $1.5 \mathrm{~mm}$ from the surface. For this plot the x-axis is again the wavelength but the y-axis is the intensity. A baseline subtraction of 550 units has been used in order to display the intensity relative to zero.

The test condition was a soft Nitrogen condition which consisted of $12.50 \mathrm{~g} / \mathrm{min} \mathrm{N}_{2}$ and $53.51 \mathrm{~g} / \mathrm{min}$ Ar. $\mathrm{CO}_{2}$ was injected with a mass flow rate of $0.69 \mathrm{~g} / \mathrm{min}$ through a FiberForm plug in the holder. The surface of the holder is at zero on the y-axis and is represented by a green line. The plasma is flowing upwards in the figure. The data was captured once the conditions reached steady state. This state was confirmed by monitoring the live data from the camera and waiting until no changes in the spectrum could be detected.
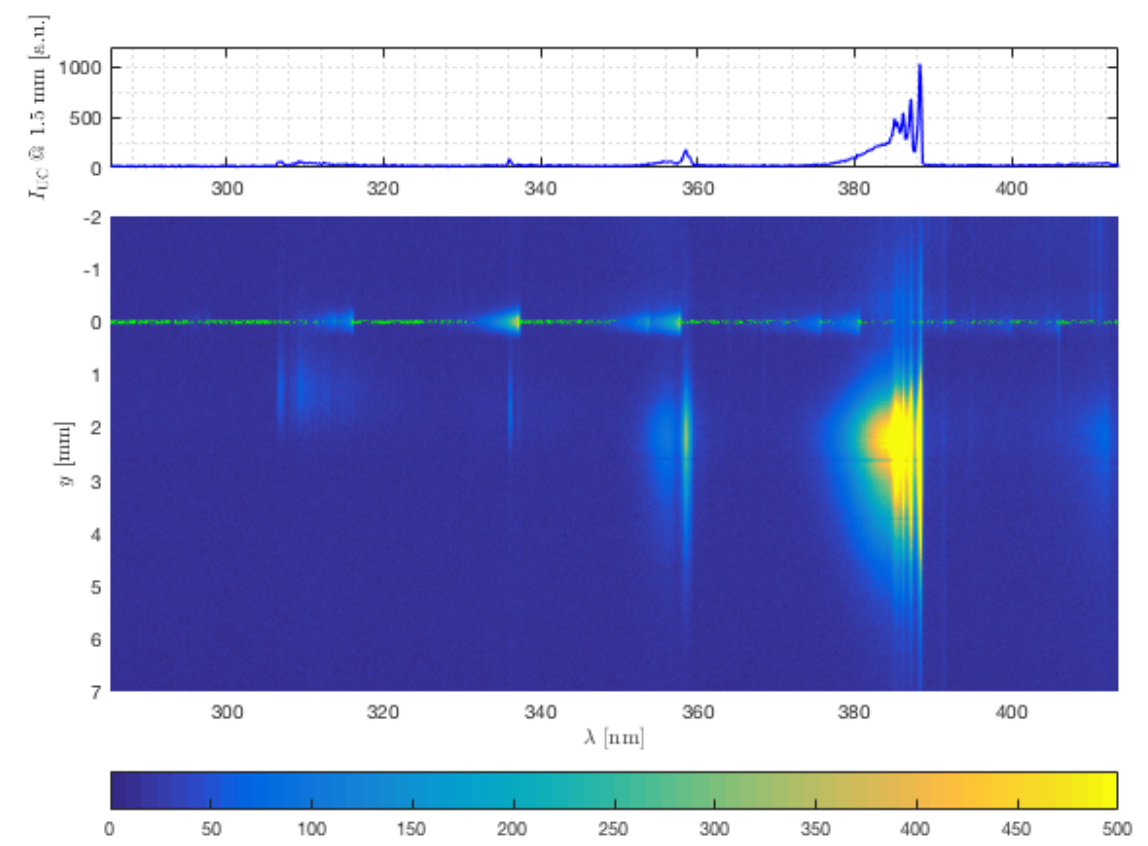

Figure 7: Single frame of injection emission taken in an AR/N2 flow.

Two CN features can be identified. The first at $\sim 358 \mathrm{~nm}$ and the second at $\sim 390 \mathrm{~nm}$. An NH feature can also be identified at $\sim 335 \mathrm{~nm}$ and an $\mathrm{OH}$ feature at $\sim 310 \mathrm{~nm}$. Due to the spatial resolution of the camera the locations of where the majority of these molecules reside can be determined. The two CN features are located mainly between 1.5 and $2.5 \mathrm{~mm}$ off the surface of the probe. This helps to confirm that the injectant gas is indeed providing a buffer layer between the probe and the plasma. The detection of NH and $\mathrm{OH}$ are also of interest in this case because hydrogen is not one of the gases being injected into the system. It is the author's opinion that the hydrogen is most likely being released by the FiberForm plug or. Figure 8 below shows a more detailed spatially resolved trace of three spectra from the above figure. The three spectra are: $\mathrm{CN}(\mathrm{B}-\mathrm{X}), \mathrm{NH}(\mathrm{A}-\mathrm{X})$ and $\mathrm{N}_{2}$, and $\mathrm{OH}(\mathrm{A}-\mathrm{X})$. 

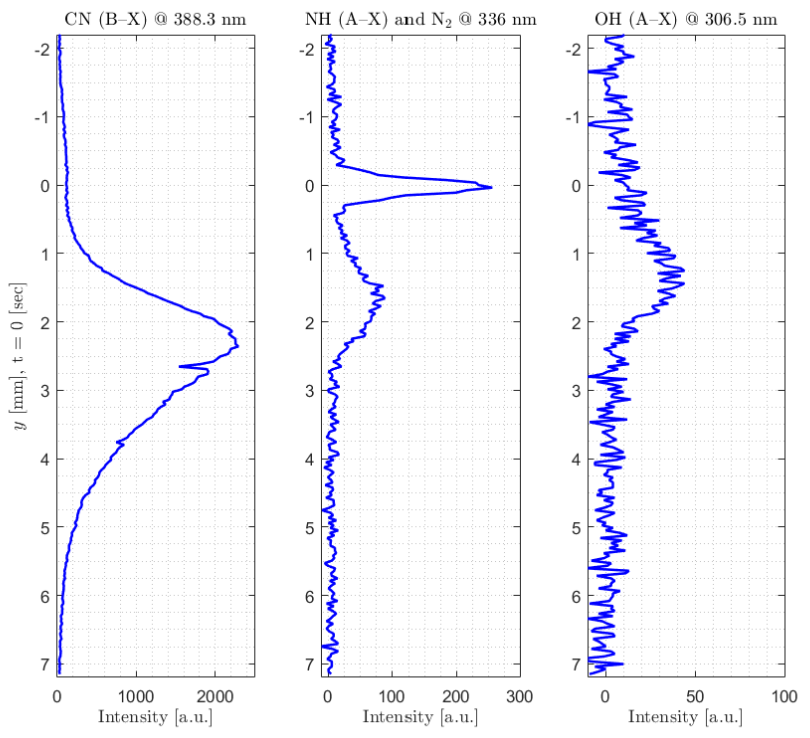

Figure 8: Spatially resolved traces of $\mathrm{CN}, \mathrm{NH}$ and $\mathrm{N}_{2}$, and $\mathrm{OH}$.

The CN trace confirms in more detail that most of the $\mathrm{CN}$ is located upstream of the surface of the injection holder. Reaffirming that $\mathrm{CN}$ does not form until it is well away from the holder face, indicating that a buffer layer is being formed by the $\mathrm{CO}_{2}$ injectant. The $\mathrm{NH}$ and $\mathrm{N}_{2}$ trace has a unique trait in that this band's spike occurs at the face of the holder. This makes it extremely simple to pinpoint the probe's surface location within the sprectra data set. The band also shows in the second spike that the interactions between the injectant gas and the plasma are occurring upstream of the sample face. The third band, the $\mathrm{OH}$ band, shows an increase in intensity slightly before the $\mathrm{NH}$ band in terms of location but has a much lower intensity. This could potentially mean that the $\mathrm{CO}_{2}$ is dissociating almost as soon as it leaves the holder and is then beginning to form $\mathrm{OH}$ with the hydrogen released by the plug.

\section{B. Computational Modeling}

The flow modeling in this work is performed by using the hypersonics CFD code, LeMANS. ${ }^{6}$ LeMANS is a finite volume multi-dimensional CFD code for simulating continuum flows with inclusion of nonequilibrium effects. In prior work, ${ }^{7}$ subsonic boundary conditions were implemented in LeMANS to properly model the conditions exhibited by the UVM ICP chamber. The boundary conditions used in the present work can be found in Figure 9. A blowing surface boundary condition is used in this work to simulate the carbon dioxide gas injection from the surface that was performed in the experiments to mimic pyrolysis gas. The experimental conditions modeled in this work can be found in Table 3.

After the flow field simulation is completed, several lines of sight are extracted and used by the nonequilibrium radiation code $\mathrm{NEQAIR}^{8}$ to generate emission spectra for comparison with experimental measurements.

\begin{tabular}{cccccc}
\hline & Inlet species & Blowing species & $\mathrm{T}_{\text {inlet }}[\mathrm{K}]$ & $\mathrm{T}_{\text {surface }}[\mathrm{K}]$ & Pressure $[\mathrm{kPa}]$ \\
\hline Case 1 & $\operatorname{Ar}(0.8919 \mathrm{~g} / \mathrm{s}), \mathrm{N}_{2}(0.2084 \mathrm{~g} / \mathrm{s})$ & $\mathrm{CO}_{2}(0.0115 \mathrm{~g} / \mathrm{s})$ & 5700 & 1000 & 21.3 \\
\hline
\end{tabular}

Table 3: Experimental conditions modeled for $\mathrm{Ar} / \mathrm{N}_{2}-\mathrm{CO}_{2}$ case. 
The species included in the simulations are: $\mathrm{Ar}, \mathrm{N}_{2}, \mathrm{~N}, \mathrm{C}, \mathrm{CN}, \mathrm{CO}_{2}, \mathrm{CO}, \mathrm{O}, \mathrm{N}_{2}^{+}, \mathrm{N}^{+}$, and $\mathrm{E}^{-}$. The chemical reaction mechanism is (reaction rates obtained from Martin et al. ${ }^{9}$ ):

$$
\begin{gathered}
\mathrm{N}_{2}+\mathrm{M} \rightleftharpoons \mathrm{N}+\mathrm{N}+\mathrm{M} \\
\mathrm{CN}+\mathrm{M} \rightleftharpoons \mathrm{C}+\mathrm{N}+\mathrm{M} \\
\mathrm{CO}_{2}+\mathrm{M} \rightleftharpoons \mathrm{CO}+\mathrm{O}+\mathrm{M} \\
\mathrm{CN}+\mathrm{O} \rightleftharpoons \mathrm{CO}+\mathrm{N} \\
\mathrm{N}+\mathrm{e}^{-} \rightleftharpoons \mathrm{N}^{+}+2 \mathrm{e}^{-} \\
\mathrm{N}+\mathrm{N} \rightleftharpoons \mathrm{N}_{2}^{+}+\mathrm{e}^{-} \\
\mathrm{N}_{2}+\mathrm{e}^{-} \rightleftharpoons \mathrm{N}+\mathrm{N}+\mathrm{e}^{-}
\end{gathered}
$$

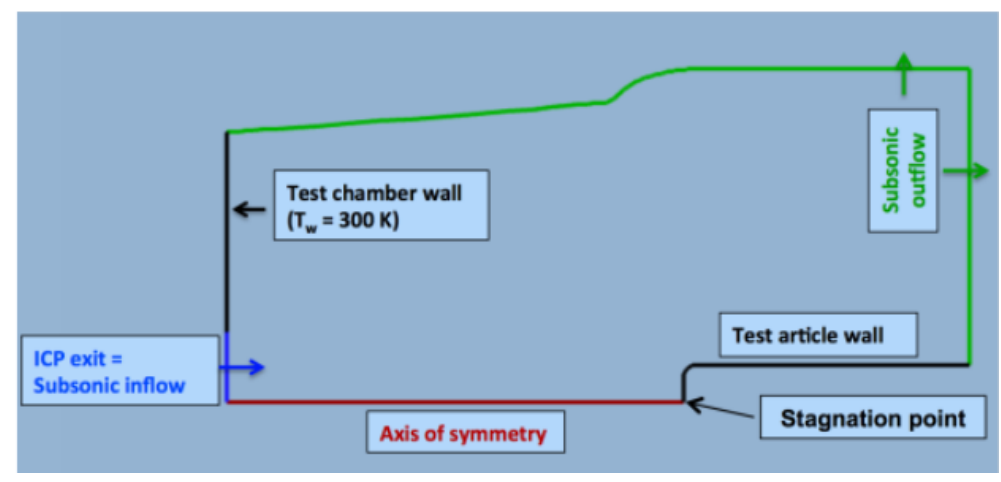

Figure 9: Boundary conditions for ICP modeling in LeMANS.

Figures 10 and 11 shows contours of translational temperature and CN number density that are generated by LeMANS. The plasma enters the ICP at the lower left corner of these images, and flows over the test sample. Figure 2 shows that elevated temperatures remain constant in the facility and only decrease due to the presence of the cooler test sample. Figure 3 illustrates the effects of the carbon dioxide that is blown from the front vertical surface of the sample. $\mathrm{CN}$ is created in a two-step process: (1) dissociation of carbon dioxide to form carbon monoxide; and (2) exchange reaction in which carbon monoxide loses an oxygen atom and gains a nitrogen atom. Figure 12 presents profiles of key species along the stagnation streamline. The sharpest increase in all species' number density can be observed near the surface. Figure 13 shows the comparison of emission spectra between the experiment and simulation. The emission spectra are calculated along a line of sight located at an axial distance of $1.5 \mathrm{~mm}$ from the frontal sample surface. The shape of the CN spectral features measured experimentally is reproduced quite well in the simulation. Note that the chemistry mechanism does not yet include $\mathrm{OH}$ and $\mathrm{NH}$, but these will be considered in the future. Figure 14 presents contours of the emission with the vertical axis representing the axial distance from the frontal sample surface. There is good correspondence with measurements with the main difference being that the peak radiation in the modeling appears to be closer to the surface than in the measurements. 


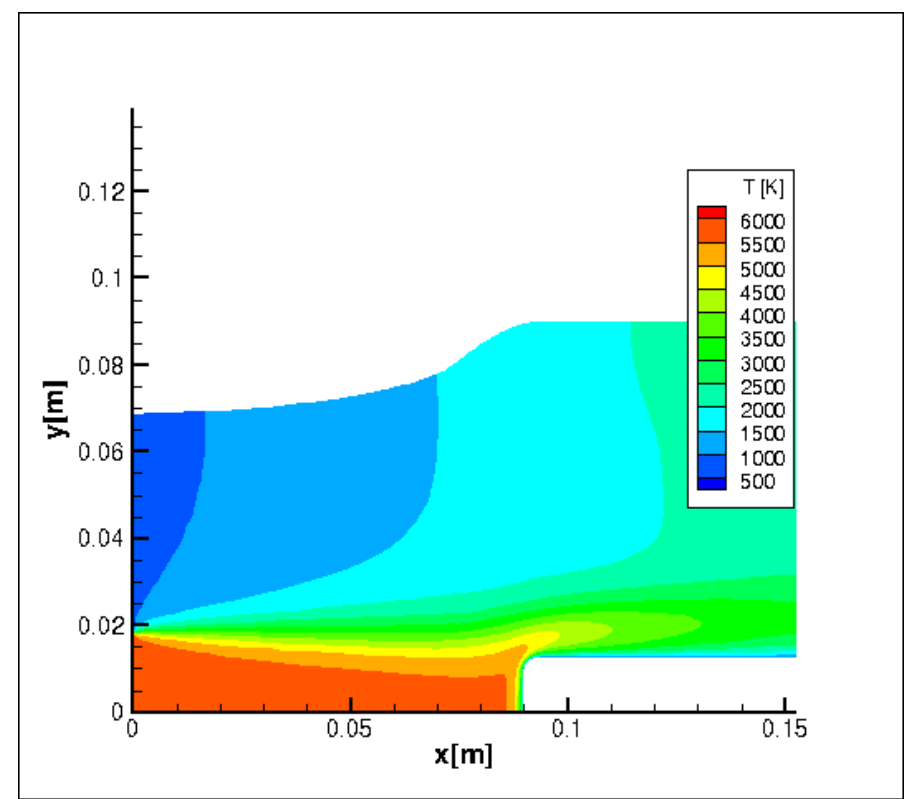

Figure 10: Contours of translational temperature.

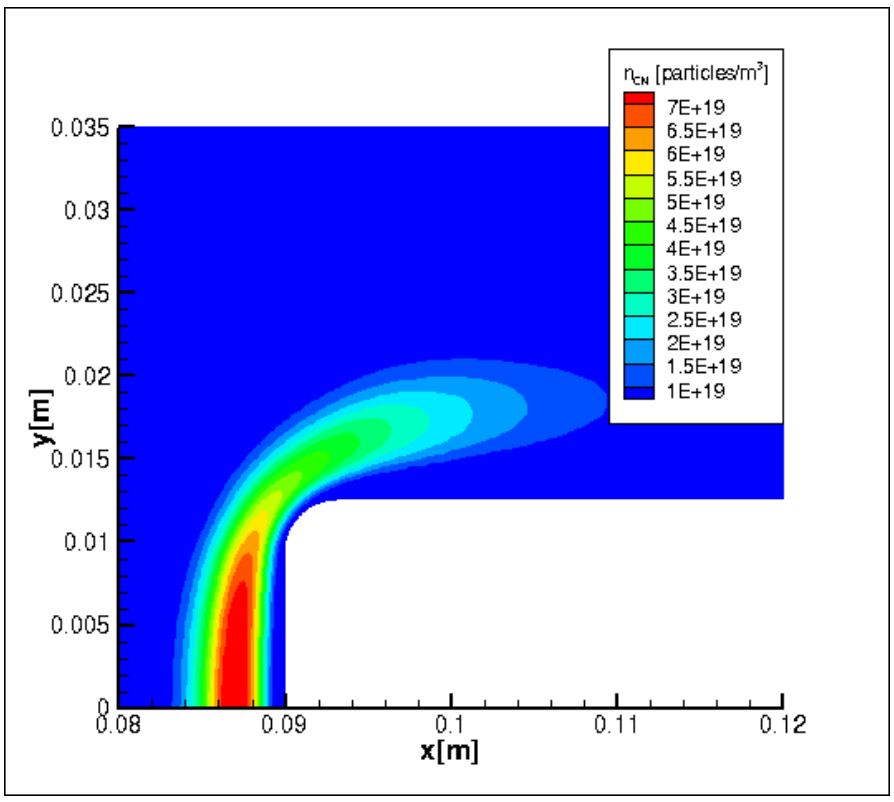

Figure 11: Contours of CN number density. 


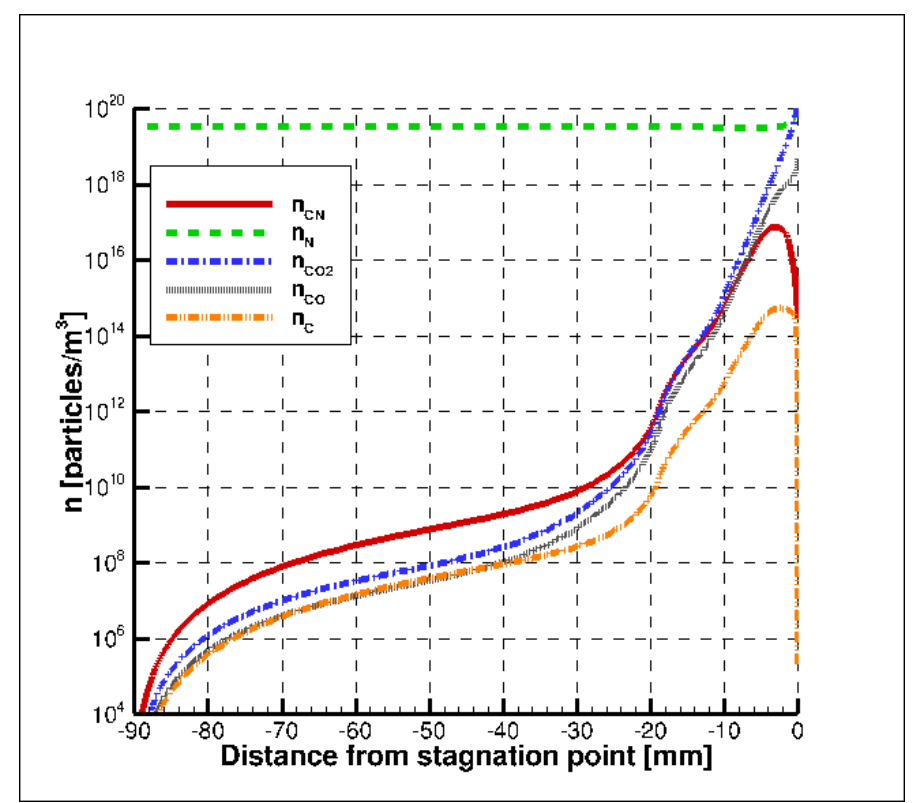

Figure 12: Key species number density along stagnation streamline.

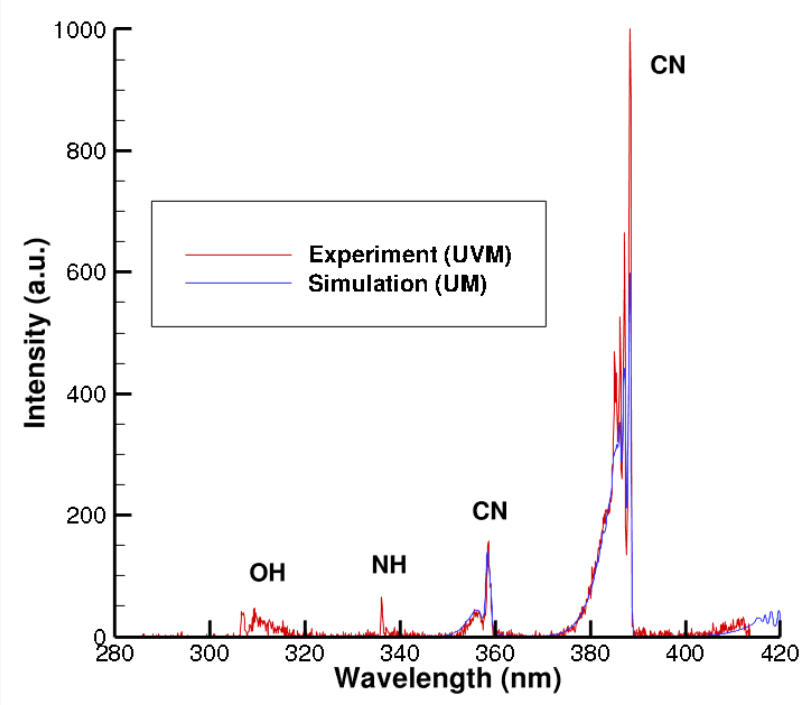

Figure 13: Comparison of emission spectra at $1.5 \mathrm{~mm}$ axial distance. 


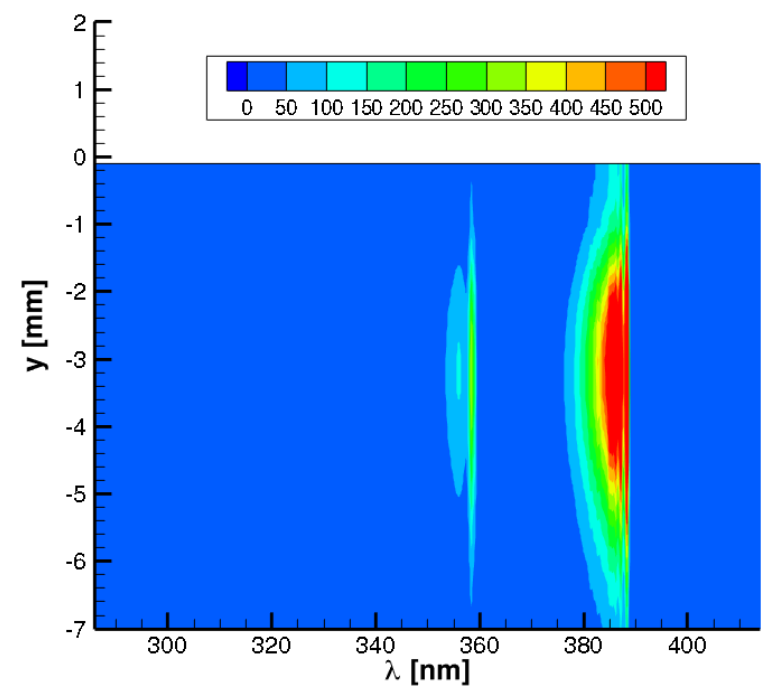

Figure 14: Calculated contours of emission (y is axial distance from frontal sample surface).

\section{Future Work}

The first attempt at this experiment has shown that there are several issues that still need to be dealt with. However, the results that were collected do look promising and have shown that the technique is sound. The work still needing to be completed is as follows.

\section{A. Probe Cooling}

It has been found that the new probe simply does not have the cooling capacity necessary to cool the holder during an injection test. Several steps have been taken to try and find a solution to this problem. A new head for an existing probe was machined and tested and found to perform slightly better but still suffered from the same problem. A different holder for the third probe in the lab has now been designed and is in the process of being finalized. Details of this probe can be found in an earlier publication from the lab. ${ }^{3}$ This design allows the cooling water to flow as close to the face of the holder as possible. This should lead to much greater cooling of the holder and therefore allow longer run times in harsher conditions.

\section{B. Surface Temperature}

As of the writing of this paper, attempts to measure the surface temperature of the face of the holder using a two-color pyrometer have been unsuccessful. This is most likely due to the luminescence of the CN layer formed. Work will be done to try and resolve this issue as well.

\section{Simulation of PICA Pyrolysis Gases}

Once a working probe has been built, focus will shift to more accurately modeling the pyrolysis gases that are released by PICA as it heats up. This work will involve designing and building the plumbing necessary to correctly mix and control the injectant gases. Once this has been completed a new test campaign will begin in order to gather and analyze the data for these conditions. 


\section{Summary}

In summary, a new probe has been designed for the UVM 30kW Inductively Coupled Plasma facility in order to help study and simulate the phenomenon of pyrolysis in phenolic impregnated carbon ablator (PICA) based heat shield materials. This probe allows for the direct injection of gas into the core of the plasma flow as well as more access to the rear of the sample for thermocouples and other measurement devices. Unfortunately, unexpected failures and problems with both the facility and the probe have caused less data to be collected than was initially expected. A single trustworthy dataset has been collected and analyzed and the results are presented above. Using the increased spatial resolution of the IsoPlane and the Pixis camera, it has been found that the buffer layer of $\mathrm{CO}_{2}$ is having a noticeable effect on the boundary layer chemistry along the stagnation line. Computational simulations of an earlier gas injection probe have been undertaken to establish a baseline configuration and an understanding of the basic injection geometry and its influence on the plasma stream. These simulations have been improved upon to more accurately model the current injection conditions. The results from the simulations have been compared to the ICP data and found to be reasonably similar in fit. Work is still being undertaken to both improve the injection mechanisms in the ICP torch as well as further refine the simulations.

\section{Acknowledgements}

Funding for this work was provided by:

NASA ESI NNX15AD78G, Scott Splinter, Technical Monitor

AFOSR FA9550-11-1-0201

\section{References}

${ }^{1}$ M. Wright, et al. "A Review of Aerothermal Modeling for Mars Entry Missions," AIAA-2010-443, 48th AIAA Aerospace Sciences Meeting Including the New Horizons Forum and Aerospace Exposition, Orlando, Florida, Jan. 4-7, 2010

${ }^{2}$ W. Owens, J. Uhl, M. Dougherty, A. Lutz and D. Fletcher, "Development of a 30kW Inductively Coupled Plasma Torch for Aerospace Material Testing," AIAA Thermophysics and Heat Transfer Conference, AIAA-2010-4322, Chicago, IL, Sep. 2010

${ }^{3}$ J. Uhl, W. Owens, J. Meyers and D. Fletcher, "Pyrolysis Simulation in an ICP Torch Facility," AIAA Thermophysics Conferece, AIAA-2011-3618, Honolulu, HI, June 2011

${ }^{4}$ C. Tillson, J. Uhl, J. Meyers, D. Fletcher, "Investigation of Pyrolysis Gas Chemistry in an Inductively Coupled Plasma Facility," 46th AIAA Thermophysics Conference, AIAA AVIATION Forum, (AIAA 2016-3235)

${ }^{5}$ Lutz, A. J., and Fletcher, D. G., "An Investigation of Non-Equilibrium Nitrogen Plasmas," 46th AIAA Plasmadynamics and Lasers Conference, AIAA Paper 2015-2961, June 2015.

${ }^{6}$ Martin, A., Scalabrin, L.C., and Boyd, I.D., "High Performance Modeling of Atmospheric Re-entry Vehicles," Journal of Physics: Conference Series, Vol. 341, 2012, Article 012002.

${ }^{7}$ Anna, A., and Boyd, I.D., "Numerical Analysis of Surface Chemistry in High-Enthalpy Flows," Journal of Thermophysics and Heat Transfer, Vol. 29, No. 4, 2015, pp. 653-670.

${ }^{8}$ Brandis, A.M. and Cruden, B.A., NEQAIRv14.0 Release Notes: Nonequilibrium and Equilibrium Radiative Transport Spectra Program, 2014.

${ }^{9}$ Martin, A., Cozmuta, I., Wright, M. J., and Boyd, I.D., "Kinetic Rates of Gas-Phase Chemistry of Phenolic-Based Carbon Ablator in Atmospheric Air," Journal of Thermophysics and Heat Transfer, Vol. 29, No. 2, 2015, pp. $222-240$. 